\title{
Effect of the addition of soluble (nutriose, inulin and polydextrose) and insoluble (bamboo, potato and pea) fibres on the quality of sugar-snap cookies
}

Running title: Effect of fibre on quality of enriched cookies

Camino M. Mancebo, Patricia Rodríguez, Mario M. Martínez, Manuel Gómez*

Food Technology Area

College of Agricultural Engineering, University of Valladolid, 34004 Palencia, Spain

Tel: +34 979-108495 fax +34 979-108302

*Corresponding author e-mail: pallares@iaf.uva.es

\begin{abstract}
Dietary fibre intake can help to improve the health of the population. Cookies are one of the most consumed bakery products. The aim of this study was to investigate the influence of different fibres on cookie quality. Soluble fibres (nutriose, inulin and polydextrose) decreased dough consistency favoured cookie spread during baking and produced wider and thinner cookies. In contrast, insoluble fibres (elongated and rounded) showed an opposite trend increasing dough consistency and giving rise to cookies with higher moisture, lower spread factor and higher hardness. Long insoluble fibres gave rise to harder cookies and with lower spread factor. Cookies made with soluble fibres were darker than the control cookie and cookies containing insoluble fibres. Therefore solubility and shape of insoluble fibres play an important role in cookie quality. Fibre selection will be key to enriched cookies development.
\end{abstract}

Keywords: Dietary fibre; rheology; biscuits; colour; cellulose. 


\section{Introduction}

It is well-known that a greater intake of dietary fibre provides a better control of blood glucose and cholesterol levels, protection against cardiovascular diseases, regulation of intestinal function and also the promotion of gut health and protection against colon cancer (Ktenioudaki and Gallagher, 2012). Fibre enrichment has been widely studied in breadmaking (Ktenioudaki and Gallagher, 2012). It has been proved that the particle size of insoluble fibres seriously affects the bread properties, with gluten (Gómez et al., 2003) or without it (Martínez et al., 2014), as well as what happens in cakes (Gómez et al., 2010). Fibre solubility or their water binding capacity, affects dough rheology and bakery product quality (Almeida et al., 2013; Martínez et al., 2014).

Cookies are a baked product, characterized by having low final water content, generally containing flour, sugar and fat as three major ingredients. Cookies, along with bread and cakes are one of the leading bakery products which are consumed in large quantities daily and they supply a convenient medium for providing dietary fibre and other healthy compounds to consumers. The increaseof dietary fibre content in cookies has been studied for different fibre sources such as resistant starch (Brennan and Samyue, 2004; Laguna et al., 2011), inulin and beta-glucan (Brennan and Samyue, 2004) or fibres from different fruits or wheat fibre (Ajila $e t$ al., 2008; Bilgiçli et al., 2007; Laguna et al., 2014) or different wheat brans (Reyes-Pérez et al., 2013; Sudha et al., 2007). However, these studies are restricted to the addition of a fibre-only type and, generally, some results are contradictory to their influence in the dough spread and final cookie texture. The reason for these contradictions may be due to differences in the formulations used, but also in the variety of fibres studied. There is an extensive literature on incorporating fibre-rich ingredients in the formulation of various types of cookies such as buriti flour (Becker et al., 2014), spirulina (Bolanho et al., 2014), or peanut skin (de Camargo et al., 2014), among others. In these studies, the effect of fibre was combined with other components, so that either can be clearly seen. It is therefore a need for larger studies where different fibres are compared with one another, and its effect on the cookie quality according to their physicochemical properties and structure. Villemejane et al. (2013) compared the effect of different brans with the effect of soluble fibres with high thickening capacity. They found that one of the factors that influenced the final cookies was the water absorption capacity of the incorporated fibres. Laguna et al. (2014) also observed differences between fibre particle size and their solubility, when comparing wheat fibre against apple fibre, with higher pectin content. Nevertheless, it is necessary to broaden studies about fibre morphology or other fibres such as soluble fibre with low thickening capacity.

The aim of this study is to analyse the effect of the addition of different fibre types according to their particle size, water absorption capacity and solubility in the dough rheology (elastic modulus, viscous modulus, complex modulus and tangent delta) and cookie quality, y in diameter and thickness, spread factor, texture and colour. The fibres used were soluble fibres (nutriose, inulin and polydextrose) and insoluble fibres (bamboo, potato and pea). 


\section{Materials and methods}

\section{Materials}

The ingredients used for cookie formulation were wheat flour supplied by Harinera Castellana S.L. (moisture content=12.88\%) (Medina del Campo, Valladolid, Spain), white granulated sugar (AB Azucarera Iberia, Valladolid, Spain), margarine $100 \%$ vegetable $(78.58 \%$ fat content) (Argenta crema, Puratos, Barcelona, Spain), sodium bicarbonate (Manuel Riesgo S.A., Madrid, Spain) and local tap water (temperature $22 \pm 2^{\circ} \mathrm{C}$ ).

The following fibres were used in this study: bamboo fibre Vitacel BAF40 (average fibre length, 50 microns) and BAF200 (average fibre length, 350 microns), potato fibre from Rettenmaier \& Sohne (Rosenberg, Germany), Nutriose ${ }^{\circledR}$ FM10 and pea fibre from Roquette Laisa (Lestrem, France), polydextrose C90 from Tate \& Lyle (London, UK) and inulin from COSUCRA Groupe Warcoing S.A. (Warcoing, Belgium).

The morphology of the fibres could be shown in (Martínez et al., 2014). Bamboo fibres (fine and coarse), mainly composed of cellulose, are thin and long fibres, each with two clearly distinct particle sizes (fibre length). Potato and pea fibres are more voluminous and irregular; potato fibre is coarser than pea fibre.

Of the soluble fibres used in this study, polydextrose shows a smaller particle size, whilst Nutriose is formed of coarser, irregular particles, some of which appear to be hollow. Inuline is more rounded and smaller than the other soluble fibres.

\section{Methods}

\section{Hydration and oil absorption properties of flour and fibres mixtures}

The mixture of the flour and the different fibres (soluble or insoluble) were characterised by their hydration and oil absorption properties.

- Water holding capacity (WHC), defined as the amount of water retained by the sample without being subjected to any stress, was evaluated by adding $100 \mathrm{~mL}$ of distilled water to $5 \mathrm{~g}( \pm 0.1 \mathrm{~g})$ of flour sample in a test tube and allowing it to hydrate for $24 \mathrm{~h}$. The hydrated solid was weighed after removing the excess water and values were expressed as grams of water per gram of solid (AACC method 88-04, 2012). Water binding capacity (WBC), or the amount of water retained by the sample after it has been centrifuged, was measured as described in AACC method 56-30.01 (AACC, 2012). Hydration properties were analysed in duplicate.

The method described by Lin et al. (1974) was used to determine oil absorption capacity (OAC). Flour $(100.0 \pm 0.2 \mathrm{mg})$ was mixed with $1.0 \mathrm{ml}$ of vegetable oil. The mixture was stirred for 1 min with a wire rod to disperse the sample in the oil. After a period of $30 \mathrm{~min}$ in the vortex mixer, tubes were centrifuged at $3000 \times \mathrm{g}$ and $4 \circ \mathrm{C}$ for $10 \mathrm{~min}$. The supernatant was carefully removed with a pipette and tubes were inverted for $25 \mathrm{~min}$ to drain the oil and the residue was weighed. The oil absorption capacity was expressed as grams of oil bound per gram of sample on dry basis. Three replicates were performed for each sample. OAC was calculated by Eq. (1): $\mathrm{OAC}(\mathrm{g} / \mathrm{g})=\mathrm{Wr} / \mathrm{Wi}$

Where $\mathrm{Wr}$ is the residue weight and $\mathrm{Wi}$ is the sample weight $(\mathrm{g}, \mathrm{db})$ 


\section{Cookie preparation}

All formulations were prepared using the same quantities of ingredients except for water, which was added to adjust dough moisture content to $15.0 \%$. The moisture content of the flour and fibre mixtures was determined as described in AACC method 44-15.02 (AACC, 2012). The following ingredients (as $\mathrm{g} / 100 \mathrm{~g}$ on dough basis) were used: flour (43.3 g/100 g), sugar (31.2 $\mathrm{g} / 100 \mathrm{~g})$, margarine $(19.4 \mathrm{~g} / 100 \mathrm{~g})$, water $(5.2 \mathrm{~g} / 100 \mathrm{~g})$ and sodium bicarbonate $(0.9 \mathrm{~g} / 100 \mathrm{~g})$. In cookies made by insoluble fibres, $15 \%$ of wheat flour was replaced by fibre. However, cookies made replacing wheat flour by soluble fibre presented too low consistency and too high stickiness of the dough. Therefore, $15 \%$ of soluble fibre addition instead replacement was preferred. The control sample was a cookie made by wheat flour without fibre.. In order to make the cookie, the margarine was heated in the microwave 1 minute at 1000 watts of powder. The margarine and sugar were then creamed at speed 4 for $180 \mathrm{~s}$ in a Kitchen Aid 5KPM50 mixer (Kitchen Aid, Michigan, USA) with a flat beater, scraping down every $60 \mathrm{~s}$. The water was then added and mixing was continued at speed 4 for $120 \mathrm{~s}$ with intermediate scraping. At the end of mixing, the cream was scraped down. Finally, flour and sodium bicarbonate were added, followed by mixing at speed 2 for $120 \mathrm{~s}$, scraping down every $30 \mathrm{~s}$. After mixing, the dough was allowed to stand for a predefined period of 30 minutes. The dough pieces were then laminated with a salva L-500-J sheeter (Salva, Lezo, Spain) (gap width $6.00 \mathrm{~mm}$ ). Cookie dough was cut with a circular cookie cutter (internal diameter, $40 \mathrm{~mm}$ ). Batches of at least 15 dough pieces were baked in an electric modular oven for 14 minutes at $185^{\circ} \mathrm{C}$. All the cookie elaborations were performed twice.

Dough rheology

The rheological behaviour of doughs was studied using a Thermo Scientific HaakeRheoStress 1 controlled strain rheometer (Thermo Fisher Scientific, Schwerte, Germany) and a Phoenix II P1$\mathrm{C} 25 \mathrm{P}$ water bath that controlled analysis temperature (set at $25^{\circ} \mathrm{C}$ ). The rheometer was equipped with parallel-plate geometry $(60-\mathrm{mm}$ diameter titanium serrated plate-PP60 Ti) with a 3-mm gap. After adjustment to the 3-mm gap, the excess dough was removed and vaseline oil (Panreac, Panreac Química SA, Castellar del Vallés, Spain) was applied to cover the exposed sample surface. During the oscillatory tests, the dough was left to rest for 800 seconds before measuring. First, a strain sweep test was performed in duplicate at $25^{\circ} \mathrm{C}$ with a stress range of 0.1-100 $\mathrm{Pa}$ at a constant frequency of $1 \mathrm{~Hz}$ to identify the linear viscoelastic region (LVR). On the basis of the results obtained, a stress value included in the linear viscoelastic region (3 to 20 $\mathrm{Pa}$ ) was used in a frequency sweep test at $25^{\circ} \mathrm{C}$ with a frequency range of $10-0.1 \mathrm{~Hz}$. Values of elastic modulus $\left(\mathrm{G}^{\prime}[\mathrm{Pa}]\right)$, viscous modulus $\left(\mathrm{G}^{\prime}\right.$ ' $\left.[\mathrm{Pa}]\right)$, complex modulus $\left(\mathrm{G}^{*}\right)\left(\mathrm{G}^{\prime}+\mathrm{i} \cdot \mathrm{G}^{\prime}\right)$ and tangent delta $(\operatorname{Tan} \delta)\left(G^{\prime} / G^{\prime}\right)$ were obtained for different frequency values $(\omega[\mathrm{Hz}])$ and analysed for $1 \mathrm{~Hz}$ (Dobraszczyk and Morgenstern, 2003). Samples were analysed in duplicate.

\section{Cookie properties}

The texture of the cookies was measured using a TA-XT2 texture analyser (Stable Microsystems, Surrey, UK) usedwith the "Texture Expert" software. Eight cookies of each elaboration were measured 24 hours after baking, using the three point bending rig probe (HDP/ 
$3 \mathrm{~PB}$ ). The experiment conditions were as follows: distance between supports $30 \mathrm{~mm}$; trigger force $5 \mathrm{~g}$, probe travel distance $30 \mathrm{~mm}$, pre-test speed $1.0 \mathrm{~mm} / \mathrm{s}$, test speed $2.0 \mathrm{~mm} / \mathrm{s}$ and posttest speed $10.0 \mathrm{~mm} / \mathrm{s}$. The maximum force $(\mathrm{N})$ and the displacement at rupture $(\mathrm{mm})$ were measured.

Sixty minutes after baking, eight cookies were weighed and their widths (diameter) and thicknesses were measured to calculate the spread factor. The diameter of each cookie was measured twice, perpendicularly, in order to calculate an average diameter. The spread factor of the cookies was calculated by dividing the average width by the thickness of the cookies. The dough of the same eight cookies was weighed before baking.

Measurements at the centre of the upper surface (crust) colour of four sugar-snap cookies from each elaboration were carried out with a Minolta CN-508i spectrophotometer (Minolta, Co. LTD, Tokyo, Japan) using the D65 illuminant with the $2^{\circ}$ standard observer. Results are expressed in the CIE L*a*b* colour space.

Statistical analysis

Differences between the parameters of the different formulations were studied by analysis of variance (ANOVA). Fisher's least significant difference (LSD) was used to describe means with 95\% confidence intervals. The statistical analysis was performed with the Statgraphics Centurion XVI software (StatPoint Technologies Inc, Warrenton, USA).

\section{Results and Discussion}

\section{Hydration properties}

Hydration properties of flour/fibre mixtures and their comparison with wheat flour are shown in table 1. In general, mixtures with insoluble fibres exhibited higher values of WBC andWHC than wheat flour, this effect being stronger in the case of potato and pea fibres. On the contrary, mixtures with fine-grained bamboo fibre did not present significant differences with wheat flour according to their values of WBC. However, their WBC values were slightly higher than those of wheat flour. Mixtures with coarse-grained bamboo fibre show high values of WHC and WBC, similar to those of pea fibre, in comparison with control sample. Eventhough the finest fibres had the highest surface contact and it is related to higher water absorption capacity (Chau et al., 2007), an opposite trend was observed in accordance with other authors (Sangnark and Noomhorm, 2003). These differences can be related to the morphology of fibres, taking into account that the bamboo fibres used were flat and lengthy, oppositeto other fibres, such as pea or potato, which are more rounded and polyhedral (Martínez et al., 2014). On the other hand, mixtures with soluble fibres showed lower values of WBC, always inferior to those of wheat flour, without presenting differences among them. Regarding WHC, these flours displayed similar values to those of wheat flour and lower to those of the majority of mixtures with insoluble fibres, which coincide with that observed by Collar et al. (2006) when comparing inulin with other fibres. With regard to OAC, the mixtures with bamboo fibres were those with higher OAC values, but in the case of the fine bamboo fibre, no significant differences with neither wheat flour nor with the rest of the mixtures with insoluble fibres were observed. 


\section{Dough characteristics}

Linear viscoelastic properties were studied by small amplitude oscillatory test (table 2). All doughs presented higher values of $G^{\prime}$ than $G^{\prime}$ ' and Tan $\delta$ values always lower than 1 , thus elastic properties prevailed against viscous ones (Lee and Inglett, 2006). A strong correlation among G', G', and G* (r values higher than 0.99) was also observed. Soluble fibre addition barely modified dough rheology, without significant differences being evident between $G$ ' and G'. Only in the case of inulin, a light increase of G', was observed, whereas nutriose and polydextrose increased the values of Tan $\delta$. These results are in agreement with the hydration properties, since soluble fibres neither modified them. On the other hand, wheat flour substitution by insoluble fibres greatly increased the values of $\mathrm{G}^{\prime}, \mathrm{G}^{\prime}$ ' andG*, and reduced the values of $\operatorname{Tan} \delta$, being this effect more important with coarse bamboo fibre, followed by potato fibre, pea fibre and less important in the case of fine bamboo fibre, which do not manage to significantly modify the values of Tan $\delta$. Laguna et al. 2014 also observed an increase in both

G' and G' values when substituted wheat flour by insoluble fibres. In table 5, a strong correlation between the rheological values and the hydration properties was found. Thereby, $\mathrm{G}^{*}$ showed a higher correlation with WHC ( $\mathrm{r}=0.96)$, likewise $\mathrm{G}^{\prime}$ and $\mathrm{G}$ ', (data not shown) whereas Tan $\delta$ showed the highest correlation with WBC. Inglett et al. (2015) already observed that adding ingredients with higher WHC, G' and G', of cookie dough were increased. Similarly, Zhang et al. (2007), studying the adequacy of different wheat cultivars in cookie-making, also observed significant correlations among the water SRC and the different rheological values. However, in the case of insoluble fibres, besides hydration properties, an influence of the particle size and geometry exists, since despite possessing similar WHC and WBC to pea and potato fibres, they gave rise to doughs with lower $G^{\prime}, G^{\prime}$ ' and $G^{*}$ than coarse bamboo fibres, lengthier and flat. Uniformly, mixtures with fine bamboo fibres, without presenting differences in their WHC values with control flour, showed much higher consistency values. Therefore, the incorporation of fibres with a lengthier shape increased the dough consistency to a greater extent compared to what their hydration properties could indicate.

\section{Cookie properties}

It is observed in table 3 that the incorporation of soluble fibres does not modify the final moisture of cookies, whereas it was increased with insoluble fibres. This effect could be attributed to their differences inwater retention. In fact, cookies made with bamboo fibre (which show lower values of WHC and WBC) presented lower moisture among insoluble fibres, observing a significant correlation $(\mathrm{r}=0.95 ; 99.9 \%)$ between the final moisture of cookies and WHC. Regarding the shape of the cookie, soluble fibres reduced the thickness of the cookie and increased its width, therefore increasing the spread factor, having a similar behaviour among them. However, insoluble fibres had the opposite effect, reducing the final width of the cookie and the spread factor. Thickness of cookies was not modified with soluble fibres, except for coarse bamboo fibre. The fibre with the most noticeable effect is coarse bamboo, followed by 
potato and pea, being the most reduced from fine bamboo fibre. During the baking process, cookie dough expands and therefore its diameter expands, until a certain time when expansion is stopped. Pareyt and Delcour (2008) indicated that the spread rate is strongly influenced by the water binding capacity, whereas Ram and Singh (2004) observed that the set time depends on the level of free water in the dough and on the dough strength. In fact, in our study, strong correlations between the geometry of doughs and the absorption properties of the mixtures, as well as their rheological properties were also observed (table 5). In general, the higher the WHC, WBC and $\mathrm{G}^{*}$ values are, the lower the final width and the spread factor and the higher the thickness is. As what occurred with the rheological values, the microstructure of the fibre seems to affect the width of the cookie, since mixtures with bamboo fibre give rise to narrower cookies with lower spread factor rather than cookies made with flours or mixtures with similar hydration properties.

All cookies enriched with fibre turned out harder (higher maximum strength) than control, but the differences were much smaller in the case of soluble fibres, which did not present significant differences between them. These results coincide with results from previous works on cookies enriched with fibres (Laguna et al., 2014; Mieszkowska and Marzec, 2016; Sudha et al., 2007). Among insoluble fibres, cookies made with coarse bamboo fibre were much harder than the rest. Cookies made with bamboo fibres also manifested higher necessary distance for the probe to break the cookie than the rest, and especially higher was that with coarser fibres. Therefore, despite the significant correlations between cookie hardness and the consistency of doughs or their shape, it seems that in this case the influence of the geometry of fibres play an important role. In fact, cookies with bamboo fibres present a higher hardness which could be expected as a function of the dough consistency or their geometry. This effect can be related to the differences in the compactness of insoluble particles in doughs. In the case of soluble fibres, differences were not observed, since they are dissolved in water and lose their original shape.

With respect to the colour, as it can be seen in table 4, and in figure 1, on the one hand, cookies with soluble fibre were darker (lower L value) than control. Probably, this fact is not because the cookie has soluble fibre, this is because the dough spread more and the exposed surface area was wider and thinner. On the other hand, cookies made with insoluble fibres were clearer than the control cookie, although only significant differences in the $\mathrm{L}$ value against control were observed in the case of coarse bamboo fibre. Cookies with soluble fibres also presented higher $a^{*}$ values, whereas cookies with bamboo fibres had the lowest $a^{*}$ values. Regarding $b^{*}$ parameter, a clear trend was not observed and only significant differences against control were observed with nutriose and polydextrose, with lower values. Our observations about the incorporation of soluble fibres are in accordance with other studies assessing the addition of similar fibres on short-dough biscuits of wheat and chickpea flour (Mieszkowska and Marzec, 2016) gluten-free breads (Martínez et al., 2014) and on cakes (Psimouli and Oreopoulou, 2012), which can be attributed to a partial hydrolysis of these compounds during baking (Korus et al., 2006). Mono and disaccharides produced in this hydrolysis would participate in Maillard reactions, generating compounds which contribute to the final colour of cookies. In cookies with 
soluble fibre, a more irregular surface with higher number of voids was also appreciated, which could be down to a higher presence of air bubbles escaping from the dough during the baking process. This explanation would also explain the lower thickness of cookies, which in turn could be related to the lower dough consistency.

\section{Conclusion}

The addition of fibre in the cookie formula modified the hydration properties, affecting the dough rheology and the cookie spread in the baking process. However, hydration properties could not explain the differences themselves. Soluble fibres decreased dough consistency favoured cookie spread during baking and produced wider and thinner cookies. In contrast, insoluble fibres showed an opposite trend increasing dough consistency and giving rise to cookies with higher moisture, lower spread factor and higher hardness. The specific length and shape of insoluble fibres added was also important, especially for texture.On the contrary, the particle length and shape of soluble fibres without thickening capacity did not affect the final result, since they were dissolved in the aqueous phase.

\section{Acknowledgements}

The authors acknowledge the financial support of the Spanish Ministry of Economy and Competitiveness (Project AGL2014-52928-C2-2-R) and the European Regional Development Fund (FEDER). The authors are grateful to Tate \& Lyle, Rettenmaier Iberia and Roquette Laisa, for supplying the raw materials. 


\section{References}

AACC International. (2012). Approved methods of the American Association of Cereal Chemists International (11 ${ }^{\text {th }}$ ed). Methods: 44-15.02 (moisture), 56-30.01 (WBC), 88-04 (WHC). St Paul, MN: American Association of Cereal Chemists.

Ajila, C.M., Leelavathi, K. \& Rao, U. (2008). Improvement of dietary fiber content and antioxidant properties in soft dough biscuits with the incorporation of mango peel powder. Journal of Cereal Science, 48, 319-326.

Almeida, E.L., Chang, Y.K. \& Steel, C.J. (2013). Dietary fibre sources in bread: Influence on technological quality. LWT - Food Science and Technology, 50, 545-553.

Becker, F.S., Damiani, C., de Melo, A.A.M., Borges, P.R.S. \& de Barros Vilas Boas, E.V. (2014). Incorporation of buriti endocarp flour in gluten-free whole cookies as potential source of dietary fiber. Plant Foods for Human Nutrition, 69, 344-350.

Bilgiçli, N., İbanoglu, Ş. \& Herken, E.N. (2007). Effect of dietary fibre addition on the selected nutritional properties of cookies. Journal of Food Engineering, 78, 86-89.

Bolanho, B.C., Egea, M.B., Morocho Jacome, A.L., Campos, I., Monteiro de Carvalho, J.C. \& Godoy Danesi, E.D. (2014). Antioxidant and nutritional potential of cookies enriched with Spirulina platensis and sources of fibre. Journal of Food Nutrition Research, 53, 171-179.

Brennan, C.S. \& Samyue, E. (2004). Evaluation of starch degradation and textural characteristics of dietary fiber enriched biscuits. International Journal of Food Properties, 7, 647-657.

Chau, C.F., Wang, Y.T. \& Wen, Y.L. (2007). Different micronization methods significantly improve the functionality of carrot insoluble fibre. Food Chemistry, 100, 1402-1408.

Collar, C., Santos, E. \& Rosell, C.M. (2006). Significance of dietary fiber on the viscometric pattern of pasted and gelled flour-fiber blends. Cereal Chemistry, 83, 370-376.

De Camargo, A.C., Vidal, C.M.M., Canniatti-Brazaca, S.G. \& Shahidi, F. (2014). Fortification of cookies with peanut skins: effects on the composition, polyphenols, antioxidant properties, and sensory quality. Journal of Agricultural and Food Chemistry, 62, 11228-11235.

Dobraszczyk, B.J. \& Morgenstern, M.P. (2003). Rheology and the breadmaking process. Journal of Cereal Science, 38, 229-245.

Gómez, M., Moraleja, A., Oliete, B., Ruiz, E. \& Caballero, P.A. (2010). Effect of fibre size on the quality of fibre-enriched layer cakes. LWT - Food Science and Technology, 43, 33-38.

Gómez, M., Ronda, F., Blanco, C., Caballero, P.A. \& Apesteguia, A. (2003). Effect of dietary fibre on dough rheology and bread quality. European Food Research and Technology, 216, 5156.

Inglett, G.E., Chen, D. \& Liu, S.X. (2015). Physical properties of gluten-free sugar cookies made from amaranth-oat composites. LWT - Food Science and Technology, 63, 214-220.

Korus, J., Grzelak, K., Achremowicz, K. \& Sabat, R. (2006). Influence of prebiotic additions on the quality of gluten-free bread and on the content of inulin and fructooligosaccharides. Food Science and Technology International, 12, 489-495. 
Ktenioudaki, A. \& Gallagher, E. (2012). Recent advances in the development of high-fibre baked products. Trends in Food Science and Technology, 28, 4-14.

Laguna, L., Salvador, A., Sanz, T. \& Fiszman, S.M. (2011). Performance of a resistant starch rich ingredient in the baking and eating quality of short-dough biscuits. LWT - Food Science and Technology, 44, 737-746.

Laguna, L., Sanz, T., Sarab, S. \& Fiszman, S.M. (2014). Role of fibre morphology in some quality features of fibre-enriched biscuits. International Journal of Food Properties, 17, 163178.

Lee, S. \& Inglett, G.E. (2006). Rheological and physical evaluation of jet-cooked oat bran in low calorie cookies. International Journal of Food Science and Technology, 41, 553-559.

Lin, M.J.Y., Humbert, E.S. \& Sosulski, F.W. (1974). Certain functional properties of sunflower meal products. Journal of Food Science, 39, 368-370.

Martínez, M.M., Díaz, Á. \& Gómez, M. (2014). Effect of different microstructural features of soluble and insoluble fibres on gluten-free dough rheology and bread-making. Journal of Food Engineering, 142, 49-56.

Mieszkowska, A. \& Marzec, A. (2016). Effect of polydextrose and inulin on texture and consumer preference of short-dough biscuits with chickpea flour. LWT - Food Science and Technology, 73, 60-66.

Pareyt, B. \& Delcour, J.A. (2008). The role of wheat flour constituents, sugar, and fat in low moisture cereal based products: a review on sugar-snap cookies. Critical Reviews in Food Science and Nutrition, 48, 824-839.

Psimouli, V. \& Oreopoulou, V. (2012). The effect of alternative sweeteners on batter rheology and cake properties. Journal of the Science of Food and Agriculture, 92, 99-105.

Ram, S. \& Singh, R.P. (2004). Solvent retention capacities of Indian wheats and their relationship with cookie-making quality. Cereal Chemistry, 81, 128-133.

Reyes-Pérez, F., Salazar-García, M.G., Romero-Baranzini, A.L., Islas-Rubio, A.R. \& RamírezWong, B. (2013). Estimated glycemic index and dietary fiber content of cookies elaborated with extruded wheat bran. Plant Foods for Human Nutrition, 68, 52-56.

Sangnark, A. \& Noomhorm, A. (2003). Effect of particle sizes on functional properties of dietary fibre prepared from sugarcane bagasse. Food Chemistry, 80, 221-229

Sudha, M.L., Vetrimani, R. \& Leelavathi, K. (2007). Influence of fibre from different cereals on the rheological characteristics of wheat flour dough and on biscuit quality. Food Chemistry, 100, 1365-1370.

Villemejane, C., Roussel, P., Berland, S., Aymard, P. \& Michon, C. (2013). Technological and sensory tools to characterize the consistency and performance of fibre-enriched biscuit doughs. Journal of Cereal Science, 57, 551-559.

Zhang, Q., Zhang, Y., Zhang, Y., He, Z.H. \& Peña, R.J. (2007). Effects of solvent retention capacities, pentosan content, and dough rheological properties on sugar snap cookie quality in Chinese soft wheat genotypes. Crop Science, 47, 656-664. 
Table 1.-Flour/fibre hydration properties and oil absorption

\begin{tabular}{lccc}
\hline Fibre type & WBC & WHC & OAC \\
\hline Control & $80.17 \mathrm{~b}$ & $6.49 \mathrm{a}$ & $1.78 \mathrm{ab}$ \\
Fine-grained bamboo & $97.32 \mathrm{c}$ & $8.42 \mathrm{a}$ & $2.10 \mathrm{bc}$ \\
Coarse-grained bamboo & $119.86 \mathrm{e}$ & $13.75 \mathrm{~b}$ & $2.15 \mathrm{c}$ \\
Potato & $106.64 \mathrm{~d}$ & $13.01 \mathrm{~b}$ & $1.82 \mathrm{ab}$ \\
Pea & $121.71 \mathrm{e}$ & $12.01 \mathrm{~b}$ & $2.02 \mathrm{abc}$ \\
Nutriose & $56.84 \mathrm{a}$ & $7.35 \mathrm{a}$ & $1.75 \mathrm{a}$ \\
Polydextrose & $55.61 \mathrm{a}$ & $7.26 \mathrm{a}$ & $1.70 \mathrm{a}$ \\
Inulin & $55.41 \mathrm{a}$ & $7.96 \mathrm{a}$ & $1.74 \mathrm{a}$ \\
Standard deviation & 27.61 & 2.88 & 0.23 \\
\hline
\end{tabular}

Values followed by the same letter in the same column are not significantly different $(\mathrm{p}<0.05)$. WBC: Water Binding Capacity; WHC: Water Holding Capacity; SV: Swelling volume; OAC: Oil absorption capacity

Table 2.- Dynamic oscillatory test results

\begin{tabular}{lcccc}
\hline Fibre type & $\mathrm{G}^{\prime}(\mathrm{Pa})$ & $\mathrm{G}^{\prime \prime}(\mathrm{Pa})$ & $\mathrm{G}^{*}$ & $\mathrm{Tan} \delta$ \\
\hline Control & $67690 \mathrm{ab}$ & $20680 \mathrm{ab}$ & $73590 \mathrm{ab}$ & $0.31 \mathrm{~cd}$ \\
Fine-grained bamboo & $152300 \mathrm{c}$ & $41845 \mathrm{~d}$ & $165900 \mathrm{c}$ & $0.28 \mathrm{c}$ \\
Coarse-grained bamboo & $389150 \mathrm{f}$ & $78820 \mathrm{~g}$ & $426250 \mathrm{f}$ & $0.20 \mathrm{a}$ \\
Potato & $279950 \mathrm{e}$ & $66825 \mathrm{f}$ & $306050 \mathrm{e}$ & $0.24 \mathrm{~b}$ \\
Pea & $228000 \mathrm{~d}$ & $54220 \mathrm{e}$ & $246700 \mathrm{~d}$ & $0.24 \mathrm{~b}$ \\
Nutriose & $66685 \mathrm{ab}$ & $23525 \mathrm{~b}$ & $73865 \mathrm{ab}$ & $0.36 \mathrm{e}$ \\
Polydextrose & $45860 \mathrm{a}$ & $18100 \mathrm{a}$ & $52035 \mathrm{a}$ & $0.40 \mathrm{f}$ \\
Inulin & $106650 \mathrm{~b}$ & $33755 \mathrm{c}$ & $119100 \mathrm{~b}$ & $0.32 \mathrm{~d}$ \\
Standard deviation & 118602 & 21840 & 129289 & 0.06 \\
\hline
\end{tabular}

Values followed by the same letter in the same column are not significantly different $(\mathrm{p}<0.05)$. G': elastic modulus; G”: viscous modulus; G*: complex modulus and Tan $\delta\left(G^{\prime \prime} / G^{\prime}\right)$ tangent delta 
Table 3.- Cookie properties

\begin{tabular}{lcccccc}
\hline Fibre type & $\begin{array}{c}\text { Cookie } \\
\text { moisture } \\
(\%)\end{array}$ & $\begin{array}{c}\text { Thickness } \\
(\mathrm{mm})\end{array}$ & $\begin{array}{c}\text { Width } \\
(\mathrm{mm})\end{array}$ & $\begin{array}{c}\text { Spread } \\
\text { ratio }\end{array}$ & $\begin{array}{c}\text { Breaking } \\
\text { strength } \\
(\mathrm{N})\end{array}$ & $\begin{array}{c}\text { Distance } \\
(\mathrm{mm})\end{array}$ \\
\hline Control & $1,95 \mathrm{a}$ & $6.32 \mathrm{~b}$ & $59.65 \mathrm{~d}$ & $9.45 \mathrm{c}$ & $21.28 \mathrm{a}$ & $0.38 \mathrm{ab}$ \\
Fine-grained bamboo & $2,91 \mathrm{~b}$ & $8.68 \mathrm{~b}$ & $50.72 \mathrm{c}$ & $5.85 \mathrm{~b}$ & $46.38 \mathrm{~d}$ & $0.56 \mathrm{c}$ \\
Coarse-grained bamboo & $3,56 \mathrm{c}$ & $11.53 \mathrm{c}$ & $42.68 \mathrm{a}$ & $3.71 \mathrm{a}$ & $140.08 \mathrm{e}$ & $1.06 \mathrm{~d}$ \\
Potato & $3,83 \mathrm{c}$ & $7.95 \mathrm{~b}$ & $47.34 \mathrm{~b}$ & $5.96 \mathrm{~b}$ & $38.50 \mathrm{c}$ & $0.43 \mathrm{~b}$ \\
Pea & $3,66 \mathrm{c}$ & $8.35 \mathrm{~b}$ & $47.44 \mathrm{~b}$ & $5.68 \mathrm{~b}$ & $44.34 \mathrm{~d}$ & $0.43 \mathrm{~b}$ \\
Nutriose & $2,10 \mathrm{a}$ & $4.42 \mathrm{a}$ & $59.42 \mathrm{~d}$ & $13.46 \mathrm{~d}$ & $28.63 \mathrm{~b}$ & $0.36 \mathrm{ab}$ \\
Polydextrose & $2,49 \mathrm{ab}$ & $4.79 \mathrm{a}$ & $63.07 \mathrm{e}$ & $13.24 \mathrm{~d}$ & $29.53 \mathrm{~b}$ & $0.35 \mathrm{a}$ \\
Inulin & $2,47 \mathrm{ab}$ & $4.92 \mathrm{a}$ & $60.64 \mathrm{~d}$ & $12.37 \mathrm{~d}$ & $30.21 \mathrm{~b}$ & $0.31 \mathrm{a}$ \\
Standard deviation & 0.11 & 2.43 & 7.44 & 3.80 & 37.15 & 0.23 \\
\hline Val
\end{tabular}

Values followed by the same letter in the same column are not significantly different $(\mathrm{p}<0.05)$.

Table 4.-Color cookie properties

\begin{tabular}{lccc}
\hline Fibre type & $\mathrm{L}^{*}$ & $\mathrm{a}^{*}$ & $\mathrm{~b}^{*}$ \\
\hline Control & $71.54 \mathrm{~b}$ & $4.41 \mathrm{c}$ & $25.45 \mathrm{bc}$ \\
Fine-grained bamboo & $77.88 \mathrm{bc}$ & $1.76 \mathrm{ab}$ & $23.58 \mathrm{bc}$ \\
Coarse-grained bamboo & $81.39 \mathrm{c}$ & $1.32 \mathrm{a}$ & $22.34 \mathrm{ab}$ \\
Potato & $71.51 \mathrm{~b}$ & $3.85 \mathrm{bc}$ & $24.43 \mathrm{bc}$ \\
Pea & $76.29 \mathrm{bc}$ & $2.98 \mathrm{abc}$ & $26.56 \mathrm{c}$ \\
Nutriose & $50.10 \mathrm{a}$ & $10.72 \mathrm{~d}$ & $19.30 \mathrm{a}$ \\
Polydextrose & $53.30 \mathrm{a}$ & $10.50 \mathrm{~d}$ & $19.63 \mathrm{a}$ \\
Inulin & $56.80 \mathrm{a}$ & $9.99 \mathrm{~d}$ & $23.07 \mathrm{~b}$ \\
Standard deviation & 11.94 & 3.95 & 2.71 \\
\hline
\end{tabular}

Values followed by the same letter in the same column are not significantly different $(\mathrm{p}<0.05)$.

Table 5.- Coefficients of correlation of the linear relationship between flour, dough and cookie properties.

\begin{tabular}{|c|c|c|c|c|c|c|c|c|c|}
\hline & WBC & OAC & $\mathrm{G}^{*}$ & $\operatorname{Tan} \delta$ & $\begin{array}{c}\text { Moisture } \\
(\%)\end{array}$ & $\begin{array}{c}\text { Thickness } \\
\text { (mm) }\end{array}$ & $\begin{array}{l}\text { Width } \\
\text { (mm) }\end{array}$ & $\begin{array}{c}\text { Spread } \\
\text { ratio }\end{array}$ & $\begin{array}{c}\text { Breaking } \\
\text { strength }(\mathrm{N})\end{array}$ \\
\hline WHC & $0.84 * *$ & & $0.96^{* * *}$ & $-0.87 * *$ & $0.95 * * *$ & $0.80^{*}$ & $-0.92 * *$ & $-0.80 *$ & \\
\hline WBC & & $0.83 *$ & $0.85^{* *}$ & $-0.93 * * *$ & $0.84 * *$ & $0.91 * *$ & $-0.95 * * *$ & $-0.97 * * *$ & \\
\hline $\mathrm{OAC}$ & & & $0.73^{*}$ & $-0.78^{*}$ & & $0.92 * *$ & $-0.85^{* *}$ & $-0.87 * *$ & $0.74^{*}$ \\
\hline $\mathrm{G}^{*}$ & & & & $-0.92 * *$ & $0.87 * *$ & $0.90 * *$ & $-0.94 * * *$ & $-0.86^{* *}$ & $0.83^{*}$ \\
\hline $\operatorname{Tan} \delta$ & & & & & $-0.80 *$ & $-0.91 * *$ & $0.95 * * *$ & $0.94 * * *$ & \\
\hline Moisture (\%) & & & & & & $0.75^{*}$ & $-0.89 * *$ & $-0.80^{*}$ & \\
\hline $\begin{array}{l}\text { Thickness } \\
(\mathrm{mm})\end{array}$ & & & & & & & $-0.93 * * *$ & $-0.96 * * *$ & $0.83^{*}$ \\
\hline Width (mm) & & & & & & & & $0.95 * * *$ & $-0.72 *$ \\
\hline Spread ratio & & & & & & & & & \\
\hline
\end{tabular}




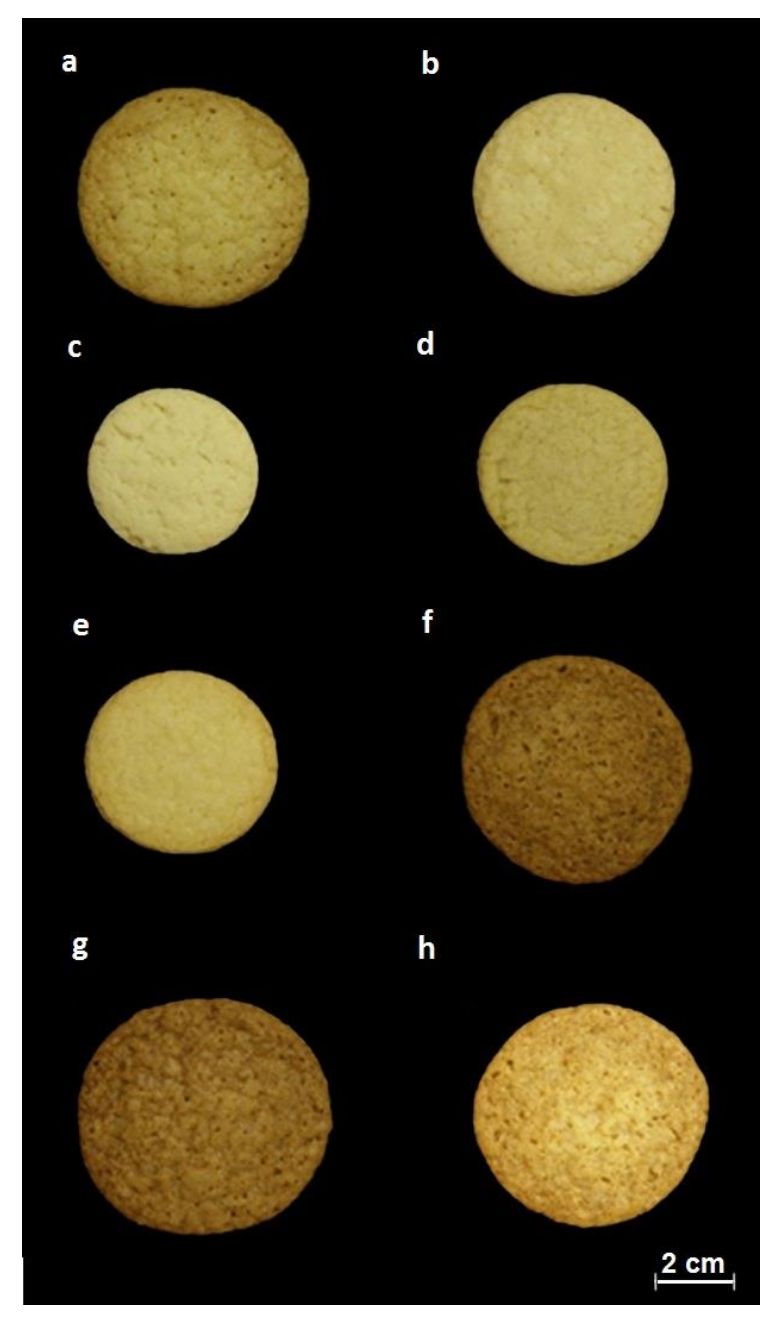

Figure 1.- Images of cookies made from wheat flour: (a) control cookie, with insoluble fibres (b) fine-grained bamboo, (c) coarse-grained bamboo, (d) potato and (e) pea and with soluble fibres (f) nutrioese, (g) polydextrose and (h) inulin. 